\title{
Determination of Tetanus Antibody Levels in Trauma Patients Referred To Shahid Beheshti Hospital in Kashan, Iran, 2014
}

\author{
Hasan Afzali ${ }^{1}$; Mohammad Reza Sharif ${ }^{2,}$; Shamsaddin Mousavi ${ }^{3}$ \\ ${ }^{1}$ Department of Infectious Diseases, Faculty of Medicine, Kashan University of Medical Sciences, Kashan, IR Iran \\ ${ }^{2}$ Trauma Research Center, Kashan University of Medical Sciences, Kashan, IR Iran \\ ${ }^{3}$ Student Research Committee, Kashan University of Medical Sciences, Kashan, IR Iran \\ *Corresponding author:Mohammad Reza Sharif, Trauma Research Center, Kashan University of Medical Sciences, Kashan, IR Iran. Tel/Fax:+98-3155620634, E-mail: mrsharifmd@yahoo.com
}

Received: January 10, 2015; Revised: July 6, 2015; Accepted: July 15, 2015

\begin{abstract}
Background: There is a noticeable difference in serologic immune status against tetanus among different age and social groups in various countries due to different national vaccination policies and methods.

Objectives: Considering that the immunization status of trauma patients against tetanus is not-known or uncertain and they may need to receive the vaccine and tetabulin, this study was conducted to determine the tetanus antibody levels in patients referred to the trauma emergency ward of Shahid Beheshti Hospital in Kashan City, Iran.

Patients and Methods: This cross-sectional study was performed on 204 trauma patients referred to the trauma emergency ward of Shahid Beheshti hospital in Kashan City, Iran, in 2014. After obtaining a written informed consent from the patients, a questionnaire consisted of demographic information and tetanus vaccination record was completed by the patients. Afterwards, a $4-5 \mathrm{~mL}$ venous blood sample was taken from each patient and the tetanus antibody level ( $\operatorname{IgG})$ was measured using the enzyme-linked immunosorbent assay method. The tetanus antibody levels equal or more than $0.1 \mathrm{IU} / \mathrm{mL}$ were considered protective. Data were analyzed using chi-square test, independent t-test and one-way ANOVA with SPSS software version 16.

Results: From a total of 204 patients, 35 cases (16.7\%) were females and 169 (83.2\%) were males with the mean age of $40.9 \pm 3.7$ years. There was no statistically significant difference in the tetanus antibody levels between both sexes $(\mathrm{P}=0.09)$. Moreover, there was no significant difference in immunization status between the patients who had a history of tetanus vaccination and those who had not received the vaccine before $(\mathrm{P}=0.67)$. The antibody levels were significantly reduced with the passage of time since the last vaccination $(\mathrm{P}<0.001)$. Also, $87.3 \%$ of the patients had the high protective level of immunity to tetanus.

Conclusions: The findings of the present study show a high level of tetanus antibody among trauma patients in this hospital; so, taking the tetanus vaccine history can be misleading. It is suggested that further studies be performed in different regions of our country and with larger sample sizes and detection of the immunization status of patients by measuring anti-tetanus antibody levels among trauma patients is recommended to make suitable policy for a national vaccine protocol in the future.
\end{abstract}

Keywords: Tetanus; Vaccination; Tetabulin; Trauma

\section{Background}

Tetanus (lockjaw) is an acute disease of the nervous system that is characterized by an acute hypertonia, generalized and severe muscle spasms and autonomic nervous system disorders. This disease is caused by a strong protein toxin called Tetanospasmin, which is produced by the anaerobic bacterium Clostridium tetani in a contaminated wound $(1,2)$. Tetanus disease can be prevented through proper immunization with the tetanus vaccine. Clostridium tetani is a Gram-positive, spore-forming anaerobic bacillus that is ubiquitous, being found throughout the world in the soil and in human and animal intestines (about 10\% of the cases). The spores are very resistant to many environmental factors, such as heat and usual antiseptics and can stay dormant for a very long time. Tetanus bacilli are found throughout the world and more prevalent in rural areas and in warm climates during summer and also in individuals whose immunization status is uncertain or incomplete. Approximately, 1,000,000 tetanus-related deaths were reported in 1980. However, the death rate related to tetanus in 2006 was reported 290,000 deaths (3-6).

There is a significant difference in serologic immune status against tetanus among different age and social groups in various countries due to different national vaccination policies and methods. A wide range of protective immunity against tetanus has been reported in different studies (7-13). In the Razaghi et al. (14) study conducted in Kashan in 2008, 35\% of the participants older than 59 years had the protective level of tetanus antibody.

In Hatamabadi et al. (15) study carried out on trauma pa-

Copyright (C) 2015, Kashan University of Medical Sciences. This is an open-access article distributed under the terms of the Creative Commons Attribution-NonCommercial 4.0 International License (http://creativecommons.org/licenses/by-nc/4.0/) which permits copy and redistribute the material just in noncommercial usages, provided the original work is properly cited. 
tients referred to emergency ward of Imam Hossein Hospital in Tehran, $80.5 \%$ of the patients had a protective level of tetanus antibody. In Dominguez et al. study in Spain in 2007 , the tetanus immunity level was $99.4 \%$ in adolescents and $68.3 \%$ in adults (16). The results of Khetsuriani et al.(17) study in Tajikistan showed that the total immunity against tetanus among children and adolescents was $78.9 \%$, and the 10 - 19 year age group had the lowest immunity. The findings of the Kurtoglu et al. study (2000 - 2001) conducted on 2465 individuals with the age range of equal or more than 6 months showed that $73.5 \%$ of the cases had complete immunity against tetanus (18). Several studies showed that the antibody response to tetanus was different in terms of age and vaccination history (19-22).

\section{Objectives}

Considering that the immunization status of trauma patients against tetanus is uncertain, and these patients may need to receive the required vaccine and tetabulin, the purpose of this study was to determine the level of tetanus antibody in trauma patients referred to the trauma emergency ward of Shahid Beheshti Hospital in Kashan, and also to evaluate the efficiency of the national protocol in preventing tetanus.

\section{Patients and Methods}

This cross-sectional study was performed on 204 trauma patients referred to the trauma emergency department of Shahid Beheshti Hospital in Kashan City, Iran, in 2014. It was postulated that the prevalence of protective immunity against tetanus in Kashan was 35\% based on the report by Razaghi et al.(14). Using the formula for estimating sample size, 204 cases were calculated as the minimum number of participants. The patients who did not consent to fill out the questionnaire were excluded from the study. The written informed consent was obtained from the patients or their family and then the participants were asked to complete a questionnaire including demographic information and tetanus vaccination records. Then, $4-5 \mathrm{~mL}$ venous blood sample was taken from the patients to perform the routine experiments and the patient's blood serum was isolated immediately and the tetanus antibody level (IgG) was measured using the Enzyme-Linked Immunosorbent Assay (ELISA) method with the IBL kit (IBL, Hamburg, Germany) and an ELISA reader machine. Based on the instructions of the IBL kit and other similar studies, the antibody levels equal or more than 0.1 were considered as optimal protective levels and the antibody levels of $0.011-0.099 \mathrm{IU} /$ $\mathrm{mL}$ were considered as low and weak immunity (23-25). This study was approved by the ethics committee of Kashan University of Medical Sciences with the approval number 1833 (date: 25/4/93).

\subsection{Data Analysis}

Data were collected, statistical tables were prepared and the descriptive indices of central tendency and dis- persion were also measured. Data were analyzed using chi-square, Independent t-test, one-way ANOVA and descriptive statistics with SPSS software version 16 (SPSS Inc., Chicago Illinois, USA).

\section{Results}

In this study, the tetanus antibody levels of trauma patients were measured. From a total of 204 patients, 35 cases $(16.7 \%)$ were females and $169(83.2 \%)$ were males with the mean age of $40.9 \pm 3.7$ years. Of 204 patients, 26 cases (12.7\%) had low levels of immunity and 178 cases (87.3\%) had high levels of immunity to tetanus. The results of the present study showed that the mean tetanus antibody level $( \pm$ SD) in male patients was $2.21 \pm 2.15$ and in females was $1.15 \pm 1.7$, which no significant difference was observed in the antibody level between males and females in this hospital $(\mathrm{P}=0.09)$.

Table 1 shows the tetanus vaccination history and duration in trauma patients. The protective tetanus antibody level was reduced with the passage of time from the last vaccination $(\mathrm{P}<0.001)$.

Table 2 shows the immunity status of vaccination groups. Results of the current study showed that there was no significant difference in the immunity status among those who had a vaccination history and those who had not $(P=0.67)$.

Table 1. Tetanus Vaccination History and Duration in Trauma Patients

\begin{tabular}{lc}
\hline Vaccine History & No. $(\%)$ \\
\hline Known duration, $\mathbf{y}$ & \\
\hline$<5$ & $52(25.4)$ \\
$5-10$ & $16(7.8)$ \\
$>10$ & $41(20.4)$ \\
\hline Total & $109(53.6)$ \\
\hline Not-remember & $82(40.1)$ \\
\hline No previous vaccination & $13(6.3)$ \\
\hline
\end{tabular}

Table 2. Immunity Status of Vaccination Groups

\begin{tabular}{lc}
\hline Vaccination Status & No. $(\%)$ \\
\hline Vaccination & $7(16)$ \\
Nonimmune & $37(84)$ \\
\hline Immune & \\
Vaccination + Tetabulin & $13(11.2)$ \\
\hline Nonimmune & $103(88.8)$ \\
Immune & \\
None & $6(13.6)$ \\
\hline Nonimmune & $38(86.4)$ \\
\hline Immune &
\end{tabular}




\section{Discussion}

This study was performed to measure the tetanus antibody level in trauma patients in Kashan City, Iran, in 2014. From a total of 204 trauma patients under the study, 35 cases (16.7\%) were males and 169 (83.2\%) were females with the mean age of $40.9 \pm 3.7$. The findings of this study revealed that the percentage of men referred to hospital due to trauma accidents is more than women and the trauma incidence in men is higher than women because men work more hours outside of the home and are at higher risk of trauma compared to women. The trauma incidence rates among men and women are in accordance with the national incidence of trauma injuries with hospitalization reported in Demographic and Health Survey in 1379 in which the incidence of trauma in men was more than three times higher than in women. In addition, the mean age of trauma patients $(40.9 \pm 3.7)$ in this study was in agreement with the national rate of mortality and inability due to trauma and shows that the selected samples, regarding their age and sex, are the appropriate samples of the population and thus can be generalized to a larger population (15). In this study, among 204 trauma patients, 26 cases $(12.7 \%)$ were not immune and $178(87.3 \%)$ had a high level of antibody and protective level of immunity. Results of the present study showed that no significant difference was observed in vaccination status between those who had received the tetanus vaccine and those who had not $(\mathrm{P}=0.67)$.

The results of a study conducted by Razaghi et al. to study the immunity status against tetanus in individuals older than 50 years in Kashan showed that the protective immunity level in patients with a history of vaccination was $57.7 \%$ and in those with no history of vaccination was $18.1 \%$, which this finding is in accordance with the finding of our study (14).

The results of the study conducted by Cassell et al. (26) in England (2002) to determine the tetanus immunization status in 269 burn patients under plastic surgery with the age range of 1 - 75 years showed that the rate of men was more than women; 200 patients had a history of vaccination, 31 cases had not received any vaccine and 38 cases were not sure whether they were vaccinated before or not. Also, results showed that the antibody level in patients who had received vaccine was significantly higher than the other patients, which is inconsistence with the results of our study (our study indicated that there was no difference with $\mathrm{P}=0.67)$. The results of the Savage EJ et al. study (2007) with the title of "Audit of tetanus prevention knowledge and practices in accident and emergency departments in England" showed that $46.2 \%$ of the patients (the majority of them) expressed that they had received the tetanus vaccine more than 10 years ago. However, no significant difference was found in tetanus antibody level among the different groups with different vaccination history; this finding is different from that of our study (27). The results of the current study showed that there was no significant difference in tetanus antibody level between male and female patients in Kashan City. A study conducted by Wu CJ et al. (2009) to study the tetanus immunity level in Taiwan showed that the protective immunity level in men (73\%) was higher than in women (49\%) (28); this finding is incongruent with that of our study, and the difference was statistically significant. In a study conducted by Gergen PJ et al. (1995), which is a population-based serologic survey of immunity to tetanus in the United States showed that the immunity level in men (79\%) was higher than in women (62.4\%) (30); this finding is not in accordance with that of our study. Razaghi et al. (2011) carried out a study about the tetanus immunization status in individuals older than 50 years in Kashan and pointed out that the immunity level in men (43.7\%) was higher than in women (27.9\%); this finding is also in accordance with those of the previous studies and unlike our study the difference was statistically significant (14).

This higher level of immunity against tetanus in men can be due to the higher prevalence rate of road-traffic accidents among men than in women and men with trauma receive tetanus booster at the time of the accident (29). Also, men receive tetanus vaccine during military service in Iran and because of this men can have higher immunity against tetanus compared to women. The results of the present study showed that $21.5 \%$ of the trauma patients had received only tetanus vaccine, $57.1 \%$ had received both tetanus vaccine and tetabulin and $21.4 \%$ had received neither vaccine nor tetabulin. The results of the study conducted by Cassell et al. in England (2002) to determine the tetanus immunization status in 269 burn patients under plastic surgery showed that from a total of 49 patients under the study, 23 cases had received the tetanus vaccine, 17 cases had received both the vaccine and tetabulin and the remainder had received neither vaccine nor tetabulin (26), which is in accordance with the results of our study. A study conducted by Frink M et al. (2006) on tetanus vaccination in the accident and emergency department in Germany reported that from a total of 155 patients, 51\% had received vaccine, $44 \%$ had received both vaccine and tetabulin and the remainder had received neither vaccine nor tetabulin (30), which its results are consistent with our results.

In conclusion, since in this study the tetanus antibody levels in three groups of patients, those who had received the vaccine, both the vaccine and tetabulin and neither the vaccine nor tetabulin, based on the national vaccination protocol are similar, this protocol has some weaknesses regarding the tetanus prevention and will need to be reviewed and updated. Since several studies showed that a rapid blood test, tetanus quick stick (TQS), could be helpful in assessing the immunity against tetanus, this test can be used in primary evaluation of trauma patients (31). Also, considering the high levels of tetanus antibody among trauma patients in this study 
and dropping of the antibody level with the passage of time from the last vaccination, it is recommended that the national vaccination schedule be carried out persistently in children and they received tetanus boosters every 10 years. Moreover, more researches with larger sample sizes should be performed and in case of obtaining similar results, the national protocol for management of the wounds be established.

\section{Acknowledgments}

We would like to thank the laboratory and emergency ward personnel of Shahid Beheshti Hospital in Kashan, Iran.

\section{Authors' Contributions}

Mohammad Reza Sharif: Study concept and design, drafting of the manuscript, critical revision of the manuscript for important intellectual content, statistical analysis, and study supervision. Hasan Afzali: Study concept and design, analysis and interpretation of data, study supervision. Shamsaddin Mousavi: Data gathering and data analysis.

\section{Funding/Support}

This study was financially supported by deputy of research at Kashan University of Medical Sciences, Kashan, Iran (Grant no 9337).

\section{References}

1. Mizuno Y, Yamamoto A, Komiya T, Takeshita N, Takahashi M. Seroprevalence of tetanus toxoid antibody and booster vaccination efficacy in Japanese travelers. J Infect Chemother. 2014;20(1):35-7.

2. Narang M, Khurana A, Gomber S, Choudhary N. Epidemiological trends of tetanus from East Delhi, India: a hospital-based study.J Infect Public Health. 2014;7(2):121-4.

3. Vollman KE, Acquisto NM, Bodkin RP. A case of tetanus infection in an adult with a protective tetanus antibody level. Am J Emerg Med. 2014;32(4):392 e3-4.

4. Fallahzadeh MK, Sajjadi S, Singh N, Khajeh M, Sagheb MM. Effect of levamisole supplementation on tetanus vaccination response rates in haemodialysis patients: a randomized double-blind placebo-controlled trial. Nephrology (Carlton). 2014;19(1):27-31.

5. Afzali H, Sharif A, Solimani Z, Razzaghi R, Heravi M. Disease's pathogens [in Persian]. Kashan: Morsal publication; 2014.

6. Mandell G, Benneth J, Dolin R. Principles and practices of infectious Diseases. 7 ed. Philadelphia: Chorchill living stone; 2015.

7. Ihara F, Otsuka Y. [Experience of two cases of tetanus without a clear history of trauma]. Nihon Jibiinkoka Gakkai Kaiho. 2014;117(1):41-5.

8. Orimadegun AE, Orimadegun BE, Adepoju AA. Immunity Against Tetanus Infection, Risk Factors for Non-Protection, and Validation of a Rapid Immunotest Kit among Hospitalized Children in Nigeria. Front Neurol. 2013;4:142.

9. Hamze M, Hlais S, Dabboussi F, Mallat H. [Anti-tetanus immunity among university students and health staff in North Lebanon and administration of anti-tetanus serums in two hospitals]. East Mediterr Health J. 2014;20(10):627-33.
10. Ang LW, James L, Goh KT. Prevalence of diphtheria and tetanus antibodies among adults in Singapore: a national serological study to identify most susceptible population groups. J Public Health (Oxf). 2015.

11. Eslamifar A, Ramezani A, Banifazl M, Sofian M, Mahdaviani FA, Yaghmaie F, et al. Immunity to diphtheria and tetanus among blood donors in Arak, central province of Iran. Iran J Microbiol. 2014;6(3):190-3.

12. Orsi GB, Modini C, Principe MA, Di Muzio M, Moriconi A, Amato MG, et al. Assessment of tetanus immunity status by tetanus quick stick and anamnesis: a prospective double blind study. Ann Ig. 2015;27(2):467-74.

13. Sung H, Jang MJ, Bae EY, Han SB, Kim JH, Kang JH, et al. Seroepidemiology of tetanus in Korean adults and adolescents in 2012.J Infect Chemother. 2014;20(7):397-400.

14. Razaghi R, khalifehsoltani A, Momenheravi M. Evaluation of tetanus Antibody in peopleswith age longer fifty years in Kashan [in Persian]. Salman J Iran. 2011;6(21):21-5.

15. HatamAbadi H, Shahi M, Sohrabi MR. Evaluation of correlation clinical enjudjment basis countries protocol with elisa test in need tetanus prevention [in persian]. Payesh J. 2010;9(1):29-37.

16. Dominguez A, Plans P, Costa J, Espunes J, Cardenosa N, Salleras L et al. The seroepidemiology of tetanus in Catalonia, Spain. Med Microbiol Immunol. 2007;196(2):115-9.

17. Khetsuriani N, Zakikhany K, Jabirov S, Saparova N, Ursu P, Wannemuehler K, et al. Seroepidemiology of diphtheria and tetanus among children and young adults in Tajikistan: nationwide population-based survey, 2010. Vaccine. 2013;31(42):4917-22.

18. Kurtoglu D, Gozalan A, Coplu N, Miyamura K, Morita M, Esen B, et al. Community-based seroepidemiology of tetanus in three selected provinces in Turkey.Jpn J Infect Dis. 2004;57(1):10-6.

19. Atkinson W. Tetanus Epidemiology and Prevention of Vaccine-Preventable Diseases. Public Health Foundation; 2015.

20. Centers for Disease Control and Prevention. Tetanus Symptoms and Complications. 2013. Available from: http://www.cdc.gov.

21. Centers for Disease Control and Prevention. Tetanus Causes and Transmission. 2013. Available from: http://www.cdc.gov.

22. Centers for Disease Control and Prevention. Tetanus For Clinicians. 2013. Available from: http://www.cdc.gov.

23. Rapisarda V, Bracci M, Nunnari G, Ferrante M, Ledda C. Tetanus immunity in construction workers in Italy. Occup Med (Lond). 2014;64(3):217-9.

24. Nemati M, Zarrin M, Mir-Abdollah SA, Rezayati MT, Mirzaee V, Bagheri A, et al. Lower serum level of anti-tetanus toxin antibodies in patients with type 2 diabetes mellitus. Acta Med Indones. 2014;46(1):44-50.

25. Filia A, Bella A, von Hunolstein C, Pinto A, Alfarone G, Declich S, et al. Tetanus in Italy 2001-2010: a continuing threat in older adults. Vaccine. 2014;32(6):639-44.

26. Cassell OC, Fitton AJ, Dickson WA, Milling MA. An audit of the tetanus immunisation status of plastic surgery trauma and burns patients. Br J Plast Surg. 2002;55(3):215-8.

27. Savage EJ, Nash S, McGuinness A, Crowcroft NS. Audit of tetanus prevention knowledge and practices in accident and emergency departments in England. Emerg Med J. 2007;24(6):417-21.

28. Wu CJ, Ko HC, Lee HC, Tsai WC, Li MG, Pao YZ, et al. Decline of tetanus antitoxin level with age in taiwan. J Formos Med Assoc. 2009;108(5):395-401.

29. Gergen PJ, McQuillan GM, Kiely M, Ezzati-Rice TM, Sutter RW, Virella G. A population-based serologic survey of immunity to tetanus in the United States. N Engl J Med. 1995;332(12):761-6.

30. Frink M, Muller CW, Ziesing S, Krettek C. [Tetanus vaccination in the accident and emergency department]. Unfallchirurg. 2006;109(11):977-82.

31. Schlumberger M, Yvonnet B, Lesage G, Tep B. Low specificity of 2 tetanus rapid tests in Cambodia. Med Mal Infect. 2015;45(12):29-33. 